JGG 2020;68:190-193

doi: $10.36150 / 2499-6564-390$

\title{
Unusual case of rosacea fulminans in older man
}

\author{
Adrian D. Meehan ${ }^{1}$, Helena Pellrud², Anna Josefson² \\ ${ }^{1}$ Department of Geriatrics, Faculty of Medicine and Health, Örebro University, Örebro, Sweden; \\ ${ }^{2}$ Department of Dermatology and Venereology, Faculty of Medicine and Health, Örebro University, \\ Örebro, Sweden
}

Rosacea fulminans (RF) is characterised by the abrupt onset of discomforting and consolidating pustules and nodules, oedema, cysts and erythema. The condition is considered rare, even rarer in men. Controversy exists whether RF should be categorised as rosacea. The aetiopathogenesis of RF remains largely unknown. We present a case of an 87-yr-old man with no previous history of rosacea or acne, admitted for a suspected systemic bacterial infection and who rapidly developed tender ocular, facial and extrafacial lesions. The patient had subfebrile temperatures, leukocytosis and moderate anaemia, but also general fatigue. The features of gender, location and extent of lesions, and biochemical response are all atypical with regards RF. The condition was successfully treated with a combination of antibiotics and oral corticosteroids. Specific management guidelines regarding RF are yet to be established, further warranting the need for continued research to determine RF's aetiology, natural course and its optimal treatment.

Key words: rosacea fulminans, ocular, extrafacial, elderly man

\section{Correspondence}

Adrian D. Meehan

Department of Geriatrics, Örebro University

Hospital, SE-701 85 Örebro, Sweden

Tel.: +46 (0)19602 2643

E-mail: adrian.meehan@regionorebrolan.se

\section{Conflict of interest}

The Authors declare no conflict of interest

\section{Funding}

None

How to cite this article: Meehan AD, Pellrud $\mathrm{H}$, Josefson $\mathrm{A}$. Unusual case of rosacea fulminans in older man. Journal of Gerontology and Geriatrics 2020;68:190-3. https://doi.org/10.36150/2499-6564-390

C Copyright by Società Italiana di Gerontologia e Geriatria (SIGG)

\section{(c) (1) () (}

\section{OPEN ACCESS}

This is an open access article distributed in accordance with the CC-BY-NC-ND (Creative Commons Attribution-NonCommercial-NoDerivatives 4.0 International) license. The article can be used by giving appropriate credit and mentioning the license, but only for non-commercial purposes and only in the original version. For further information: https://creativecommons.org/licenses/by-nc-nd/4.0/deed.en

\section{INTRODUCTION}

Rosacea fulminans (RF) is an uncommon condition typically characterised by the rapid appearance of consolidating pustules and nodules, oedema, cysts and sensitive erythematous eruptions restricted primarily to facial areas ${ }^{1}$. The condition most often occurs in younger women where it is thought that hormonal factors may have an implicatory role. Fewer than 9\% (12 individuals) of RF cases have been described in males and these cases are more frequently reported in older males ${ }^{2}$. The aetiology of RF is largely unknown, with reports suggesting that bacterial and inflammatory conditions being instrumental in the development of RF. This has made the classification of RF particularly challenging. We present a case of RF in an elderly man who was admitted to hospital for a suspected systemic bacterial infection.

\section{CASE DESCRIPTION}

An 87-year-old man, with no other reported dermatological conditions apart from having been operated for a malign melanoma from the dorsal trunk (2002) and a basalioma on his nose (2011), was admitted to our Acute Geriatric Unit (AGU) with a suspected systemic bacterial infection with no 
clear focus. Previously the patient had lived alone, had lived a physically active life and was totally independent without any apparent need of municipal care provision. The patient had recently been discharged from a Pulmonary Ward at our hospital where he had received care for progressive lethargy and markedly reduced appetite. The patient had a malignant prostate cancer but regular PSA monitoring confirmed the cancer to be latent and entirely stabile. CT-thorax had revealed a low attenuated solitary nodule in the right apical lung and it was deemed adequate with further radiological assessment in six months. At discharge, the patient had a $\mathrm{CRP}=100 \mathrm{mg} / \mathrm{l}$, but otherwise clinically stable. After only a few days at a municipal rehabilitation unit his condition deteriorated significantly, and he was then re-admitted to hospital and placed at the AGU. At admission, it was noted that he had distinct, demarcated erythema on both cheeks. The patient was clearly lethargic, had a febrile response while receiving paracetamol regularly. The initial work-ups revealed an increase in WBC $=13.3$ $\times 10^{-9} / \mathrm{l}$ and $\mathrm{CRP}=206 \mathrm{mg} / \mathrm{l}$, and even moderate anaemia with $\mathrm{Hb}=87 \mathrm{~g} / \mathrm{L}$ (Tab. I). During the following days, the patient rapidly developed skin eruptions primarily on the cheeks (Fig. 1A), eyelids, left ear canal, left shoulder, dorsal side of right wrist and a large suspected sanguinary pustule appeared on the dorsal side of digit III of the right hand. In addition, he developed distinct areas of erythema on the right wrist, left shoulder, and upper part of the chest. Substantial, bilateral periorbital oedema and signs of conjunctivitis were evident, and the patient complained of blurred vision. The eruptions, particularly in the facial area, caused considerable discomfort.

Bacterial and viral swabs and cultures were taken, initially with the suspicion of a systemic bacterial infection. Intravenous treatment with piperacillin-tazobactam was initiated and valaciclovir was given concomitantly in a suitable dosage for a herpes infection. All cultures were negative apart for a positive urine culture specimen reporting the growth of Staphylococcus ludgenesis $\left(10^{6} \mathrm{CFU} / \mathrm{ml}\right)$ (Tab. I). Complementary radiology was inconclusive. Consultants from the Infectious Diseases, Ophthalmology, and Dermatology Clinics assessed the patient. The ophthalmologist detected a limited corneal defect with fluorescein staining in his left eye, and his sight was worse in this eye which could also have been explained by a pre-existing cataract. Multiple ulcerous sores with abundant purulent secretion developed around the eyes. Biopsies from these areas revealed both actinic keratosis (right eye) and post-inflammatory changes (left eye), but no signs of malignancy. Limited madarosis was evident. The dermatologist (HP, coauthor) took a $3 \mathrm{~mm}$ biopsy from the left cheek which demonstrated ruptured folliculitis, demodex, perifollicular inflammation, and capillary telangiectasia (Fig. 2AB). The patient began immediately with a daily course of Prednisolone $30 \mathrm{mg}$ and after ten days, when the pathological examination confirmed the diagnosis of $\mathrm{RF}$, a course of tetracycline (limecycline) was initiated concomitantly. Prednisolone was slowly phased out but tetracycline was continued until follow-up.

Table I. Characteristics of and important laboratory details of the onset of rosacea fulminans (RF) in an 87-year-old man.

\begin{tabular}{|c|c|}
\hline Clinical variable/characteristic & Outcome \\
\hline Radiology & $\begin{array}{l}\text { CT-thorax \& abdomen: low attenuated solitary nodule in right apical lung } \\
\text { CT-brain: older, small CVL lesions }\end{array}$ \\
\hline Medical history & $\begin{array}{l}\text { Appendectomy, operated total arthroplasty bilaterally, BPH, excision BCC nose tip, excision MM dorsal } \\
\text { trunk }\end{array}$ \\
\hline Laboratory testing & $\mathrm{Hb}=87, \mathrm{WBC}=13.3$ (neutro $=10.8$ ), $\mathrm{CRP}=206, \mathrm{ALT}=0.69, \mathrm{AST}=0.56, \mathrm{ALP}=3.3, \mathrm{Temp} .=38.1$ \\
\hline Bacterial and viral swabs and cultures & $\begin{array}{c}\text { M.pneumoniae DNA - negative } \\
\text { C.pneumoniae DNA - negative } \\
\text { H.simplex } 1,2-\text { negative } \\
\text { V.Zoster DNA - negative } \\
\text { Nasal cavity, conjunctival \& ulcer swabs - negative } \\
\text { Blood cultures taken in } 4 \text { bottles - negative } \\
\text { Urine culture - Staphylococcus ludgenesis }\left(10^{6} \mathrm{CFU} / \mathrm{ml}\right)\end{array}$ \\
\hline RF duration before initial assessment & 2-3 days \\
\hline Head and neck locations & Cheeks, eyelids, left ear \\
\hline Other anatomical sites & Left shoulder, upper anterior thorax, dorsal right wrist, distal phalangeal joint dig. III dx \\
\hline Morphology & Pustules, papules, nodules (right wrist), erythema, oedema, no comedones \\
\hline Histopathology & $\begin{array}{c}3 \mathrm{~mm} \text { biopsy left cheek - ruptured folliculitis, demodex (folliculorum?), } \\
\text { perifollicular inflammation, telangiectasia }\end{array}$ \\
\hline
\end{tabular}

CVL: cerebrovascular lesion; BPH: benign prostate hyperplasia; BCC: basal cell carcinoma; MM: malign melanoma; Hb: haemoglobin; WBC: white blood cells; CRP: creactive protein; ALT: alanine transaminase; AST: aspartate transaminase; ALP: alkaline phosphatase; CFU: colony-forming unit 


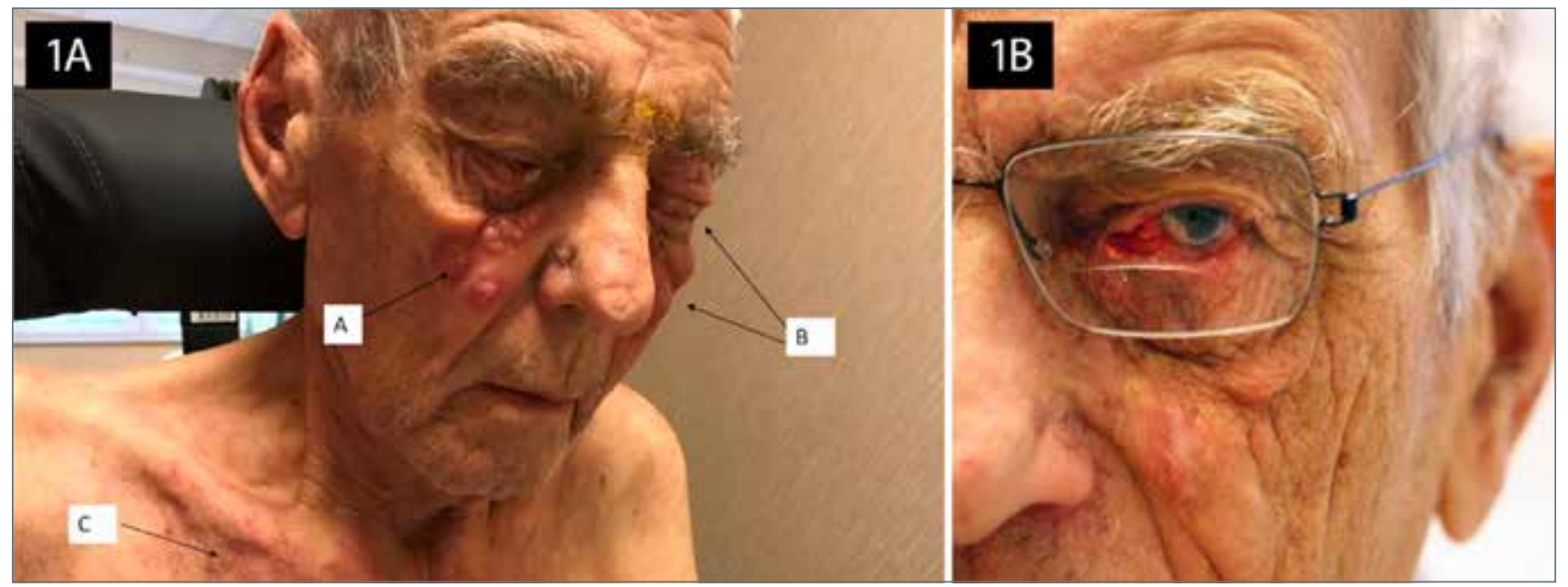

Figure 1. A. Abrupt presentation at day 3 of facial and extrafacial skin eruptions in an 87-year-old man: a) Pustules on both cheeks, b) Pustules and nodules on cheek and eyelid. Bilateral periorbital oedema. Blurred vision and initially suspected conjunctivitis, c) Erythema and discrete nodules in a distinct area of the upper chest; B. 87-year-old man at 3 months follow-up revealing in principle complete dermatological remission, though slight erythema slight exists around the left eye.

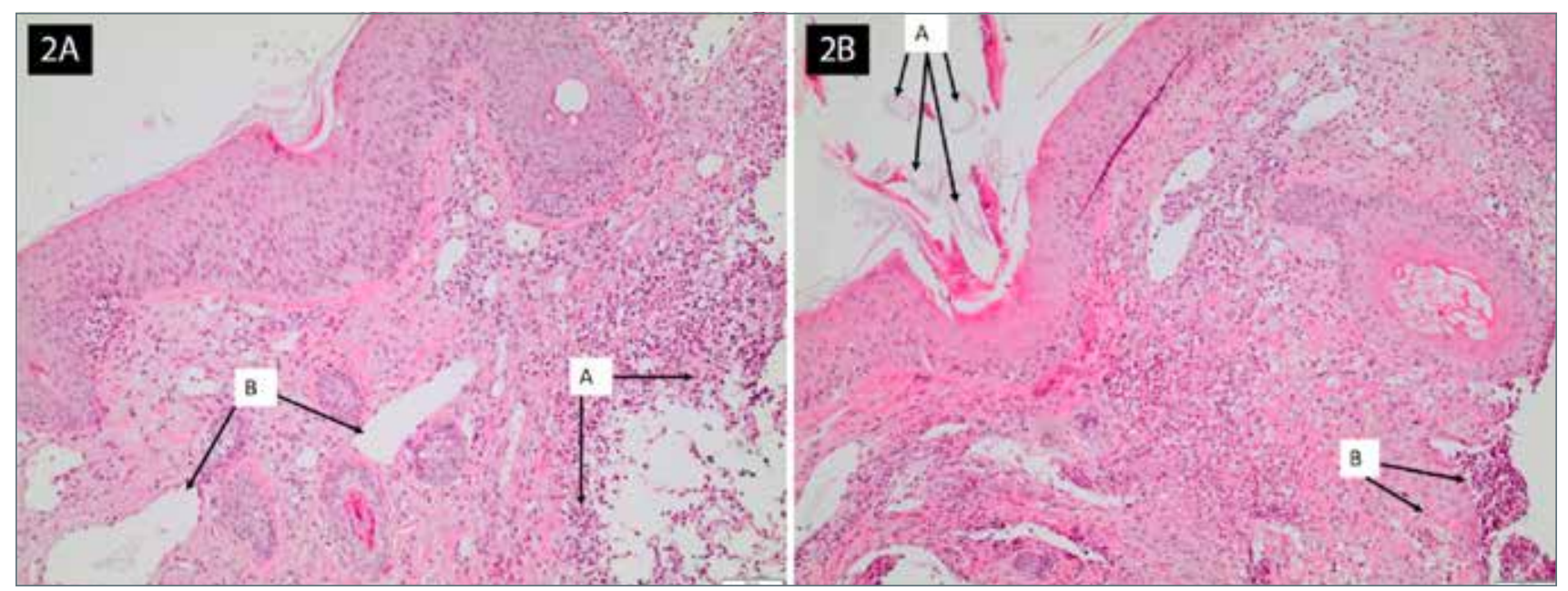

Figure 2. A. Skin biopsy from left cheek showing: a) perifollicular inflammation with infiltration of neutrophils and lymphocytes in the dermis, and b) telangiectasia; B. Skin biopsy from left cheek showing a) demodex, and b) ruptured folliculitis, abnormal inflammation with infiltration of neutrophils and lymphocytes in the dermis.

Isotretinoin was considered as an alternative if the initiated treatment was inadequate. The patient's general physical health improved and could soon be discharged for rehabilitation. At three months follow-up, the patient was in complete remission with regards skin complaints, though slight scarring had occurred on the left cheek. Discrete erythema remained around the left eye (Fig. 1B).

\section{DISCUSSION}

Rosacea fulminans (RF) is a rare condition and was first described by O'Leary and Kierland as a separate condition which they termed "pyoderma faciale" ${ }^{3}$. Plewig et al. reported the positive effects of cortisone and isotretinoin in a series of twenty patients and thus suggested the condition not to be a genuine pyoderma but rather a variant of rosacea, proposing the new term of RF ${ }^{4}$.

The pathogenesis of RF is hitherto unknown, with the current understanding of the condition being fragmentary and its classification contentious ${ }^{1,5}$. Though the condition is rare, most of the approximately 140 cases described in the literature in English involve fertile women who are otherwise healthy, characterised by the 
rapid debut of large coalescent nodules, pustules, erythema and oedema ${ }^{2,4-7}$. As such, medication (e.g. contraceptives) and hormonal factors may have relevance for the condition's development, but even inflammatory and vascular factors have in addition been described as having a contributory role. It has even been speculated that emotional and diverse stress events - as in the suspected systemic infection in the elderly man described in this article (the stress event being an infection of unknown aetiology) - may give rise to RF ${ }^{2}$. Conflicting evidence exists as to whether patients typically have a history of rosacea and acne ${ }^{2,8}$. The current case differs from most cases in a number of features: gender, age (though in males, an older age of onset is more typical), extrafacial symptoms, and the relatively uncommon feature of ocular involvement (however ocular rosacea is described as a subtype) ${ }^{6}$.

The treatment prescribed to the current patient is in line with the review of Walsh et al. which revealed that the most common treatment of RF is oral antibiotics, oral isotretinoin and systemic corticosteroids ${ }^{2}$. The majority of reports confirm the advantageous use of corticosteroids. Improvement often occurs rapidly within a matter of weeks, or a few months, and recurrence is rare. Refractory cases may be treated with isotretinoin or, in some cases, adjuvant treatment with dapsone ${ }^{6,9}$. A clear causal correlation between RF and infection has yet to be established, which possibly confirms the initial observations of Plewig et al. that RF is not a true pyoderma ${ }^{2,4,7}$.

\section{CONCLUSIONS}

In conclusion, we present a further case of RF in an older male which illustrates some features which are typical and others unusual in this somewhat rare condition. Whether RF is a true variant of rosacea remains a subject of discussion. Clinical assessment can often initially be challenging, and while the current patient responded well to antibiotics and steroids, there seems to be important individual differences in the clinical presentation of RF suggestive of the possibility that various forms of severity exist at group level. Specific management guidelines regarding RF are yet to be established, further warranting the need for continued research of this condition to determine RF's aetiology, natural course and its optimal management.

\section{Consent}

Informed consent was received from the patient for whom identifying information was used in this article.

\section{Acknowledgements}

Our sincere thanks to Dr. Luiza Dorofte, Dept. of Pathology at University Hospital in Örebro, for assistance with the development of biopsy images.

\section{References}

1 Mori R MS, Honda T, Horiguchi Y, et al. Chronic superficial pyoderma of the face. J Dermatology 2005;32:189-92.

2 Walsh RK, Endicott AA, Shinkai K. Diagnosis and treatment of rosacea fulminans: a comprehensive review. Am $\mathrm{J}$ Clin Dermatol 2018;19:79-86. https://doi.org/10.1007/ s40257-017-0310-0

3 O'Leary P, Kierland R. Pyoderma faciale. Arch Dermatol 1940;41:451-62.

4 Plewig G, Jansen T, Kligman AM. Pyoderma faciale. A review and report of 20 additional cases: is it rosacea? Arch Dermatol 1992;128:1611-7. https://doi.org/10.1001/ archderm.128.12.1611

5 Koh KY NS, Tan WP. Rosacea fulminans. Indian J Dermatol 2014;80:272-4.

6 Kim TG, Noh SM, Do JE, et al. Rosacea fulminans with ocular involvement. Br J Dermatol 2010;163:877-9. https:// doi.org/10.1111/j.1365-2133.2010.09880.x

7 Sasaki H, Otsuka A, Iga N, et al. A case of rosacea fulminans staining negative for LL-37. Eur $\mathrm{J}$ Dermatol 2016;26:101-2. https://doi.org/10.1684/ejd.2015.2671

8 Selden S. Pyoderma faciale. J Am Acad Dermatol 2005;53:1104-5; author reply 5-6. https://doi. org/10.1016/j.jaad.2005.08.030

9 Bormann G, Gaber G, Fischer M, et al. Dapsone in rosacea fulminans. J Eur Acad Dermatol Venereol 2001;15:465-7. https://doi.org/10.1046/j.1468-3083.2001.00325.x 